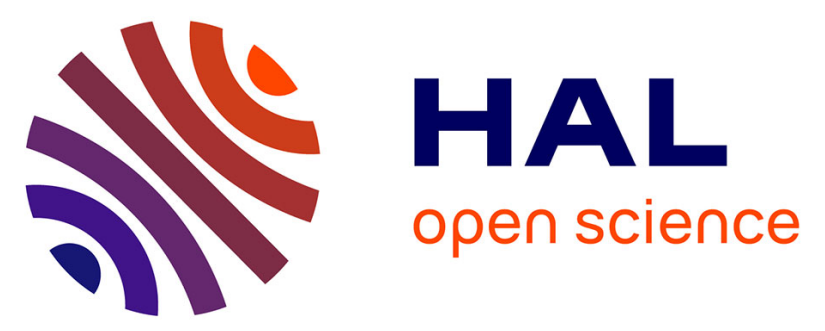

\title{
Efficient and accurate calculation of band gaps of halide perovskites with the Tran-Blaha modified Becke-Johnson potential
}

Boubacar Traoré, Gaëlle Bouder, William Lafargue-Dit-Hauret, Xavier Rocquefelte, Claudine Katan, Mikaël Kepenekian, Fabien Tran

\section{To cite this version:}

Boubacar Traoré, Gaëlle Bouder, William Lafargue-Dit-Hauret, Xavier Rocquefelte, Claudine Katan, et al.. Efficient and accurate calculation of band gaps of halide perovskites with the Tran-Blaha modified Becke-Johnson potential. Physical Review B, 2019, 99 (3), pp.035139. 10.1103/PhysRevB.99.035139 . hal-01982557

\section{HAL Id: hal-01982557 https://hal.science/hal-01982557}

Submitted on 15 Jan 2019

HAL is a multi-disciplinary open access archive for the deposit and dissemination of scientific research documents, whether they are published or not. The documents may come from teaching and research institutions in France or abroad, or from public or private research centers.
L'archive ouverte pluridisciplinaire HAL, est destinée au dépôt et à la diffusion de documents scientifiques de niveau recherche, publiés ou non, émanant des établissements d'enseignement et de recherche français ou étrangers, des laboratoires publics ou privés. 
B. Traore et coll. Phys. Rev. B 2019; https://journals.aps.org/prb/accepted/ ad074O6bJf21963f606384008ec0d981852de2b59

\title{
Efficient and accurate calculation of band gaps of halide perovskites with the Tran-Blaha modified Becke-Johnson potential
}

\author{
Boubacar Traore \\ Univ. Rennes, ENSCR, INSA Rennes, CNRS, \\ ISCR (Institut des Sciences Chimiques de Rennes) \\ - UMR 6226, F-35000 Rennes, France* and \\ Univ Rennes, INSA Rennes, CNRS, \\ Institut FOTON - UMR 6082, F-35000 Rennes, France \\ Gaëlle Bouder, William Lafargue-Dit-Hauret, Xavier \\ Rocquefelte, Claudine Katan, and Mikaël Kepenekian \\ Univ. Rennes, ENSCR, INSA Rennes, CNRS, \\ ISCR (Institut des Sciences Chimiques de Rennes) - UMR 6226, F-35000 Rennes, France* \\ Fabien Tran \\ Institute of Materials Chemistry, Vienna University of Technology, \\ Getreidemarkt 9/165-TC, A-1060 Vienna, Austria
}




\begin{abstract}
We report on a reoptimization of the Tran-Blaha modified Becke-Johnson (TB-mBJ) potential dedicated to the prediction of the band gaps of 3-dimensional (3D) and layered hybrid organicinorganic perovskites (HOP) within pseudopotential-based density functional theory methods. These materials hold promise for future photovoltaic and optoelectronic applications. We begin by determining a set of parameters for 3D HOP optimized over a large range of materials. Then we consider the case of layered HOP. We design an empirical relationship that facilitates the prediction of band gaps of layered HOP with arbitrary interlayer molecular spacers with a computational cost considerably lower than more advanced methods like hybrid functionals or GW. Our study also shows that substituting interlayer molecular chains of layered HOP with Cs atoms is an appealing and cost-effective route to band gap calculations. Finally, we discuss on the pitfalls and limitations of TB-mBJ for HOP, notably its tendency to overestimate the effective masses due to the narrowing of the band dispersions. We expect our results to extend the use of TB-mBJ for other low-dimensional materials.
\end{abstract}




\section{INTRODUCTION}

Three dimensional hybrid halide organic-inorganic perovskites (HOP) of general formula $\mathrm{ABX}_{3}$, where $\mathrm{A}$ is an organic or inorganic cation, $\mathrm{B}$ a divalent metal cation and $\mathrm{X}$ a halide anion, are at the center of extensive worldwide research on the quest of promising materials for future photovoltaic and optoelectronic applications. The main reason is the spectacular rise of their power conversion efficiency from $3.8 \%^{1}$ to over $23 \%^{2}$ in less than a decade, already surpassing the efficiency of polycrystalline Si-based solar cells. Moreover, they are fabricated using low cost solution process methods and present interesting features in lightemission $^{3,4}, \mathrm{X}$-ray detection ${ }^{5}$ and lasing ${ }^{6}$. On the flip side, they suffer from instabilities when exposed to lighting and humidity conditions. To circumvent these instabilities, layered halide perovskites have gained renewed interest because of their resistance to the above-mentioned stringent operating conditions ${ }^{7,8}$. In addition, they present attractive optoelectronic properties related to their large exciton binding energies ${ }^{9}$ and multi-excitonic resonances ${ }^{10}$ as a result of quantum and dielectric confinement effects ${ }^{11,12}$. Naturally, the appearance of such interesting properties requires a joint experimental and theoretical effort to understand their fundamental photophysical origins.

On the theoretical side, Kohn-Sham (KS) density functional theory (DFT), ${ }^{13,14}$ within the local density approximation (LDA) ${ }^{14}$ or the generalized gradient approximation (GGA), ${ }^{15,16}$ has been widely used to study the optoelectronic properties of hybrid halide perovskites and has proved to be efficient to compute materials structure-property relationships at a reasonable computational $\operatorname{cost}^{17,18}$. However, it is well known that the standard LDA and GGA are not appropriate to compute the band gap since giving too small band gaps or even a wrong metallic behavior rather than an insulating one in some cases (see, e.g., refs 19 and 20). Let us note that in the case of Pb-based HOP, a fortuitous agreement between DFT and experimental band gaps is obtained if spin-orbit coupling (SOC) effect is not considered ${ }^{21}$.

For solids, different levels of theory exist to improve the prediction of band gaps such as hybrid functionals ${ }^{22-25}$ and the more well founded quasi-particle correction method within the many body $G W$ approximation ${ }^{26,27}$. Hybrid functionals, which combine Hartree-Fock exchange and LDA/GGA exchange-correlation, have been used to improve the band gap of $\mathrm{HOP}^{8,28,29}$. Similarly, the $G W$ approximation has also been adopted for these materials ${ }^{30-34}$. In general, the computational cost of the hybrid and GW methods are several orders of 
magnitude higher than LDA/GGA. Although calculations with hybrid functionals are computationally more affordable as compared to $G W$, both methods remain heavy for HOP especially the layered ones, which even in their very simplest structures easily exceed hundreds of electrons. This prompts the need of adopting computationally cheaper methods and yet accurate enough to predict the band gap of HOP.

Among the affordable band gap correction schemes (see refs 35 and 36 for comparative studies), there is the Tran-Blaha modified Becke-Jonhnson (TB-mBJ) potential ${ }^{37}$, which has proven to be as accurate as hybrid functionals or $G W$ for band gap prediction, but at a cost which is of the same order of magnitude as with LDA/GGA functionals. It has been quite successful for a variety of materials ranging from classical semiconductors, like $\mathrm{Si}$ or Ge, to strongly correlated transition-metal oxides (e.g., $\mathrm{FeO}$ or $\mathrm{NiO}$ ) and rare-gas solids ${ }^{35-40}$. The TB-mBJ method, which consists of only a potential (no energy functional) combines a modified version of the BJ exchange potential ${ }^{41}$ with LDA for correlation ${ }^{42,43}$. The TB-mBJ exchange potential reads

$$
v_{x, \sigma}^{\mathrm{TB}-\mathrm{mBJ}}(\mathbf{r})=c v_{x, \sigma}^{\mathrm{BR}}(\mathbf{r})+(3 c-2) \frac{1}{\pi} \sqrt{\frac{5}{6}} \sqrt{\frac{t_{\sigma}(\mathbf{r})}{\rho_{\sigma}(\mathbf{r})}}
$$

where $v_{x, \sigma}^{\mathrm{BR}}$ is the Becke-Roussel (BR) potential ${ }^{44}$ designed to model the Coulomb potential created by the exchange hole, $\rho_{\sigma}=\sum_{i=1}^{N_{\sigma}}\left|\psi_{i, \sigma}\right|^{2}$ is the spin- $\sigma$ electron density and $t_{\sigma}=$ $(1 / 2) \sum_{i=1}^{N_{\sigma}} \nabla \psi_{i, \sigma}^{*} \cdot \nabla \psi_{i, \sigma}$ is the kinetic-energy density. The parameter $c$ in Eq. (1) is given by

$$
c=\alpha+\beta\left(\frac{1}{V_{\text {cell }}} \int_{\text {cell }} \frac{\left|\nabla \rho\left(\mathbf{r}^{\prime}\right)\right|}{\rho\left(\mathbf{r}^{\prime}\right)} d^{3} r^{\prime}\right)^{1 / 2}
$$

where $V_{\text {cell }}$ is the unit cell volume, and $\alpha=-0.012$ and $\beta=1.023 \mathrm{bohr}^{1 / 2}$ are the parameters that were determined from least square fitting of the experimental band gaps of a set of solids (see ref $^{37}$ for details). As discussed in refs ${ }^{35,36,38}$ the TB-mBJ potential owes its success for band gaps to two of its ingredients, namely, $t_{\sigma}$ (which makes TB-mBJ a meta-GGA potential) and the average of $|\nabla \rho| / \rho$ in the unit cell in Eq. (2).

Moreover from Eq. (2), one can see that $\beta$ determines the sensitivity of $c$ with respect to the inhomogeneity in the electron density in the unit cell (measured as the average of $|\nabla \rho| / \rho$ in the unit cell). With respect to the term containing beta, the first term in $c$ (i.e., $\alpha)$ is just a shift.

Recently, Jishi et al. ${ }^{45}$ applied the TB-mBJ method to lead halide HOP using the all- 
electron WIEN2k code ${ }^{46}$ and reported an improvement of the band gaps with respect to the standard GGA/PBE functional of Perdew et al. ${ }^{16}$ However, since the agreement with experiment was still not satisfying (underestimation of the order of $\sim 1 \mathrm{eV}$ ), Jishi et al. reoptimized the parameters $\alpha$ and $\beta$ in Eq. (2) in order to further improve the results. With their new values for $\alpha$ and $\beta$, the agreement with experiment was then excellent (error of $\sim 0.15 \mathrm{eV}$ maximum). These refined parameters should, to some extent, be transferable to other perovskites.

However, on the technical side, it is important to underline that the parameters of Jishi et $a l$., as well as the original ones ${ }^{37,47}$, were determined using an all-electron code. This means that these parameters are in principle not valid with DFT codes using pseudopotentials, since $c$ depends on the average of $|\nabla \rho| / \rho$ which should (strongly) depend on the type of electron density $\rho$ (all-electron or pseudo). Therefore, reoptimizing $\alpha$ and $\beta$ for a pseudopotentialbased DFT code (as used in the present work) is necessary and can be appealing for larger benchmark studies. Furthermore, to our knowledge there is no report of the performance of TB-mBJ on layered hybrid halide perovskites or any other layered materials in the literature. Such a performance assessment would be beneficial to contextualize the adequacy of such a cheap band gap correction DFT scheme in studying the optoelectronic properties of large systems, such as layered HOP, with pseudopotential codes.

In this contribution, we report on the reoptimization of the TB-mBJ parameters for 3-dimensional (3D) and layered halide perovskites within pseudopotential-based implementation of DFT as realized in ABINIT ${ }^{48}$. As already mentioned, SOC plays a major role in HOP and is systematically taken into account in the present work. Since layered HOP are generally large systems, we propose a scheme that involves the substitution of the interlayer molecules with Cs atoms ${ }^{49-51}$ which reduces the computational burden with negligible loss of accuracy. Furthermore, such a substitution would be beneficial for larger benchmarking of layered HOP using TB-mBJ. From the evolution of the optimized $c$ parameter in Eq. (2) with the interlayer spacing of layered HOP, we propose an empirical relationship that links these two quantities so as to facilitate the choice of $c$ for an arbitrary layered HOP. We also consider the effect of the TB-mBJ potential on the effective masses and show that they are largely overestimated especially for layered HOP as compared to available experimental values. Finally, we discuss on the pitfalls and limitations of TB-mBJ when applied to layered hybrid halide perovskites. We believe that such considerations will be useful for other 
low-dimensional materials.

\section{COMPUTATIONAL DETAILS}

The pseudopotential DFT calculations were done with the ABINIT code ${ }^{48}$. The relativistic, norm-conserving, separable, dual-space Gaussian-type pseudopotentials of Hartwigsen, Goedecker, and Hutter $(\mathrm{HGH})$ were used for all atoms ${ }^{52}$. More specifically, we considered $1 s^{1}, 2 s^{2} 2 p^{2}, 2 s^{2} 2 p^{3}, 3 s^{2} 3 p^{5}, 4 s^{2} 4 p^{5}, 5 s^{1}, 5 s^{2} 5 p^{5}, 6 s^{1}, 5 s^{2} 5 p^{2}$ and $6 s^{2} 6 p^{2}$ as valence electrons for $\mathrm{H}, \mathrm{C}, \mathrm{N}, \mathrm{Cl}, \mathrm{Br}, \mathrm{Rb}, \mathrm{I}, \mathrm{Cs}, \mathrm{Sn}$ and $\mathrm{Pb}$, respectively. The all-electron calculations were done with the WIEN2k code, ${ }^{46}$ which is based on the linearized augmented plane-wave method. ${ }^{53,54}$ The LDA ${ }^{14,42,43}$ and TB-mBJ ${ }^{37}$ methods were used for the exchange-correlation effects. TB-mBJ in abinit was invoked from the library of exchange-correlation functionals Libxc $^{55}$. All the calculations were performed taking into account spin-orbit coupling (SOC)

effects. The experimental crystal structures without any geometry optimization were used for all calculations. The following structures were considered with the space group provided in parenthesis:

3D systems: $\mathrm{MAPbI}_{3}(\mathrm{I} 4 / \mathrm{mcm})^{56}$, where $\mathrm{MA}=\mathrm{CH}_{3} \mathrm{NH}_{3}, \mathrm{MAPbI}_{3}(\mathrm{Pnma})^{57}, \mathrm{MAPbBr}_{3}$ $(\mathrm{Pm}-3 \mathrm{~m})^{58}, \mathrm{MAPbBr}_{3}(\mathrm{Pnma})^{59}, \mathrm{CsPbI}_{3}(\mathrm{Pnma})^{60}, \mathrm{CsPbBr}_{3}(\mathrm{Pnma})^{61}, \mathrm{CsPbCl}_{3}$ (Pm$3 \mathrm{~m})^{62}, \mathrm{RbPbI}_{3}(\mathrm{Pnma})^{60}, \mathrm{MASnI}_{3}(\mathrm{I} 4 \mathrm{~cm})^{63}, \mathrm{CsSnI}_{3}(\mathrm{Pnma})^{64}$.

2D systems: $\left(\mathrm{C}\left(\mathrm{NH}_{2}\right)_{3}\right)\left(\mathrm{CH}_{3} \mathrm{NH}_{3}\right) \mathrm{PbI}_{4}(\mathrm{Imma})^{65},\left(\mathrm{C}_{m} \mathrm{H}_{2 m+1} \mathrm{NH}_{3}\right)_{2} \mathrm{PbI}_{4}(\mathrm{Pbca})^{66,67}$ with $m=4,6$ and 10 .

\section{RESULTS AND DISCUSSION}

\section{A. 3D HOP compounds}

We start with the reoptimization of the TB-mBJ parameters $\alpha$ and $\beta$ in Eq. (2) for perovskites in the framework of a pseudopotential method. To do this, we selected a wide range of $3 \mathrm{D} \mathrm{ABX}_{3} \mathrm{HOP}$ as listed in Table I. In the course of the optimization procedure, we observed that it is enough to vary $\alpha$, while keeping $\beta$ fixed to its original value $\left(1.023 \mathrm{bohr}^{1 / 2}\right)^{37}$. Hence, we optimized $\alpha$ to predict the band gap of a particular 3D perovskite (e.g. $\mathrm{MAPbI}_{3}$ ). Subsequently, we applied those reoptimized TB-mBJ parameters to the other HOP struc- 
tures. The new set of values that we propose is given by

$$
\alpha=0.65, \quad \beta=1.023 \mathrm{bohr}^{1 / 2} .
$$

We note that our new value for $\alpha$ is much closer to the value 0.4 by Jishi et $a l .{ }^{45}$ optimized for lead halide HOP than the original TB-mBJ value (-0.012). Furthermore, the value for $\beta$ optimized by Jishi et al. is $1.0 \mathrm{bohr}^{1 / 2}$, which is very close to $1.023 \mathrm{bohr}^{1 / 2}$. Table I compares the TB-mBJ band gaps calculated using our reoptimized pseudopotential (pp) parameters (ppTB-mBJ) and the values $\alpha=0.4$ and $\beta=1.0 \mathrm{bohr}^{1 / 2}$ of Jishi et al. (jTBmBJ). For comparison purpose, LDA results are also shown. It is clear from Table I that since the parameters of Jishi et al. were determined using all-electron densities, they are not optimal within a pseudopotential framework. Indeed, jTB-mBJ underestimates by a sizeable amount, 0.4-0.7 eV, the band gap of all HOP, while, as already mentioned, Jishi et al. obtained excellent agreement with experiment with the all-electron WIEN2k code. However, the prediction becomes much better with our reoptimized ppTB-mBJ values, since the errors are below $0.2 \mathrm{eV}$ in all cases. Noteworthy, the same set of ppTB-mBJ parameters works very well for both $\mathrm{Pb}$ and $\mathrm{Sn}$ based $\mathrm{HOP}$, as well as for different halide anions, thus showing the generality of ppTB-mBJ for 3D HOPs.

We remind that our predicted band gaps do not take into account the effects of band gap renormalization due to electron-phonon coupling in HOP, which has been shown to be non-negligible especially at high temperature ${ }^{68-70}$. With $\mathrm{CsSnI}_{3}$ for instance, band gap renormalization was calculated to be $+0.11 \mathrm{eV}$ at $300 \mathrm{~K}^{68}$. From Table I, the measurement temperature does not exceed room temperature and we hypothesize that our predicted ppTB-mBJ band gaps would remain in reasonable agreement with experiment should there be any correction due to band gap renormalization. Concerning LDA, the underestimation of the band gap is substantial in all cases and varies from $\sim 1 \mathrm{eV}$ (for $\mathrm{MAPbI}_{3}$ ) to $\sim 2.5 \mathrm{eV}$ (for $\mathrm{CsPbCl}_{3}$ ). In the case of $\mathrm{CsSnI}_{3}, \mathrm{LDA}$ gives a band gap of nearly zero, while experiment indicates a values of $1.3 \mathrm{eV}$. Again, our ppTB-mBJ parameters lead to fair agreement with experiment.

Regarding the shape of the bands, Figure 1 compares the band structures in the case of $\mathrm{MAPbI}_{3}$ (Pnma) and $\mathrm{CsSnI}_{3}$ (Pnma) using LDA and ppTB-mBJ. In these systems, the band gap is direct at $\Gamma$. With $\mathrm{MAPbI}_{3}$, the spin-orbit coupling (SOC) induces a larger splitting at the conduction band minimum (CBM) which amounts to 0.77 and $0.61 \mathrm{eV}$ 
TABLE I: Band gap (in eV) of 3D HOP obtained with LDA and two reoptimized versions of TB-mBJ: jTB-mBJ from Jishi et al. ${ }^{45}$ and ppTB-mBJ proposed in the present work. The space group is indicated in parenthesis. SOC effects were included in all calculations.

\begin{tabular}{lcccc}
\hline \hline 3D HOP & LDA & jTB-mBJ & ppTB-mBJ & Exp. \\
\hline $\mathrm{MAPbI}_{3}$ (I4/mcm) & 0.44 & 1.05 & 1.43 & $1.48-1.61^{71-75 \mathrm{a}}$ \\
$\mathrm{MAPbI}_{3}$ (Pnma) & 0.44 & 1.26 & 1.64 & $1.65^{76 \mathrm{~b}}$ \\
$\mathrm{MAPbBr}_{3}$ (Pm-3m) & 0.22 & 1.58 & 2.16 & $2.18-2.22^{74,77,78 \mathrm{c}}$ \\
$\mathrm{MAPbBr}_{3}$ (Pnma) & 0.64 & 1.98 & 2.43 & - \\
$\mathrm{CsPbI}_{3}$ (Pnma) & 1.71 & 2.87 & 3.33 & $3.17^{79 \mathrm{~d}}$ \\
$\mathrm{CsPbB}_{3}$ (Pnma) & 0.48 & 1.85 & 2.45 & $2.25^{61,80 \mathrm{c}}$ \\
$\mathrm{CsPbl}_{3}$ (Pm-3m) & 0.47 & 2.10 & 2.84 & $2.86^{80 \mathrm{c}}$ \\
$\mathrm{RbPbI}_{3}$ (Pnma) & 1.71 & 2.83 & 3.30 & $3.14^{81 \mathrm{~d}}$ \\
$\mathrm{MASnI}_{3}$ (I4cm) & 0.16 & 0.89 & 1.55 & $1.2-1.4^{63 \mathrm{e}}$ \\
$\mathrm{C}_{\mathrm{C} S \mathrm{M}_{3} \text { (Pnma) }}$ & 0.03 & 0.81 & 1.21 & $1.30^{64 \mathrm{c}}$ \\
\hline \hline
\end{tabular}

for LDA and ppTB-mBJ, respectively. For both compounds, a rather obvious qualitative difference between LDA and ppTB-mBJ is the width of the valence band maximum (VBM) and CBM that is smaller with ppTB-mBJ. According to the study of Waroquiers et al. ${ }^{82}$, the TB-mBJ potential deteriorates the agreement with experiment for the band width. The implication of the band dispersions on the effective masses will be discussed later. Also shown in Figure 1, are one of the components of the complex spinor wave functions of the 
VBM and CBM at the $\Gamma$ point. We recover the well-known hybridization pattern namely: the VBM is composed of an anti-bonding hybridization between $\mathrm{Pb}(\mathrm{Sn})-6 \mathrm{~s}(5 \mathrm{~s})$ and I-5p states, while the CBM consists mainly of a bonding hybridization between $\mathrm{Pb}(\mathrm{Sn})-6 \mathrm{p}(5 \mathrm{p})$ and $\mathrm{Pb}(\mathrm{Sn})-6 \mathrm{p}(5 \mathrm{p})$ states, which is in agreement with previous works ${ }^{21,50}$.
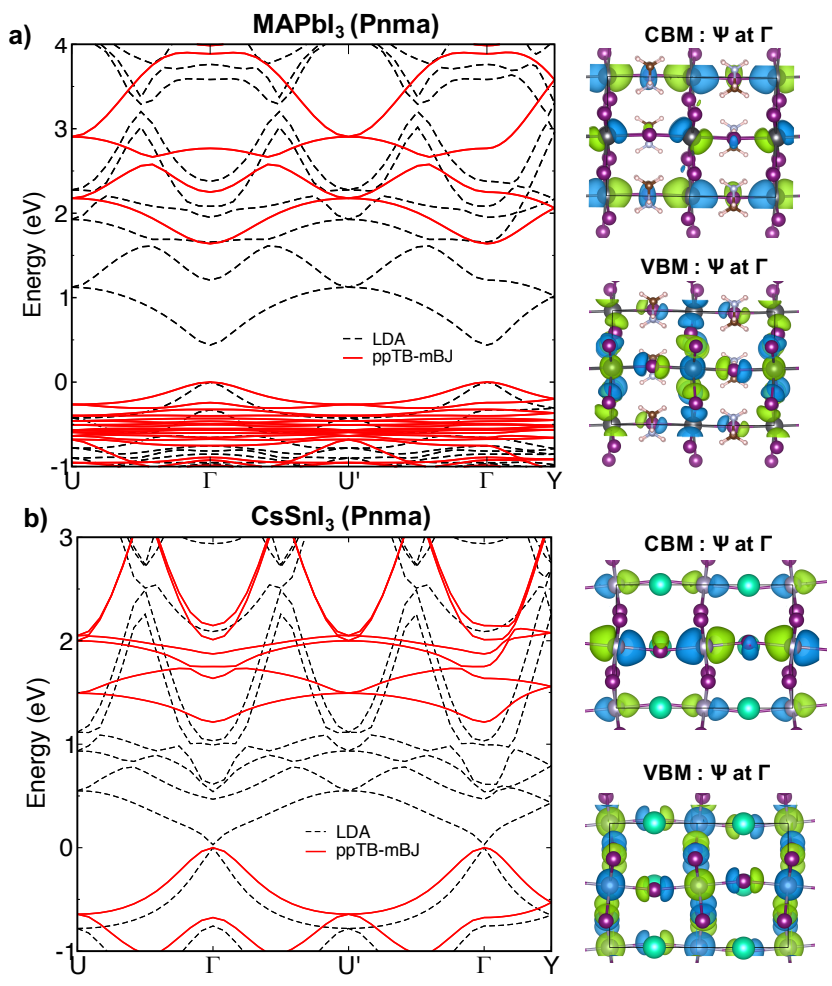

FIG. 1: Electronic structure of $\mathrm{MAPbI}_{3}$ and $\mathrm{CsSnI}_{3}$ in their orthorhombic phases. Left: comparison of LDA and ppTB-mBJ band structures of a) $\mathrm{MAPbI}_{3}$ and b) $\mathrm{CsSnI}_{3}$. Right: wave functions (one of the complex spinor components) at the VBM and CBM calculated using ppTB-mBJ for a) $\mathrm{MAPbI}_{3}$ and b) $\mathrm{CsSnI}_{3}$.

\section{B. 2D Layered HOP}

In this section, we inspect the suitability of the reoptimized TB-mBJ potential proposed above, ppTB-mBJ, to predict the band gap of layered 2D HOP. Figure 2a shows the considered experimental 2D HOP structures. They consist of the guanidinium-methylammonium lead iodide perovskite structure $\left(\mathrm{C}\left(\mathrm{NH}_{2}\right)_{3}\right)\left(\mathrm{CH}_{3} \mathrm{NH}_{3}\right) \mathrm{PbI}_{4}{ }^{65}$, hereafter referred to as GAMA (where $\mathrm{GA}=\mathrm{C}\left(\mathrm{NH}_{2}\right)_{3}$ and $\mathrm{MA}=\mathrm{CH}_{3} \mathrm{NH}_{3}$ denote guanidinium and methylammonium, 
respectively) and three members ( $m=4,6$ and 10) of the alkylammonium lead iodide $\left(\mathrm{C}_{m} \mathrm{H}_{2 m+1} \mathrm{NH}_{3}\right)_{2} \mathrm{PbI}_{4}$ series (hereafter called $\mathrm{C} m$ where $m$ is the number of carbon atoms in the alkyl chain $)^{66,67}$. The self-assembled nature of layered HOP is reminiscent of a heterostructure and can be conceptualized as composite materials, where one can define a pseudo inorganic layer with the interlayer molecular cations replaced by $\mathrm{Cs}^{+51}$. Such a substitution with Cs atoms is appealing since it reduces the computational load while having little effect on the electronic structure near the band edge states as shown earlier ${ }^{49,51}$. The validity of this substitution when using the ppTB-mBJ potential is discussed in Figure S1 of the supplementary material (SM) ${ }^{83}$.

Let us note that in hybrid perovskites, room temperature cubic and tetragonal 3D systems are averaged structures because of the dynamical motion of the organic cations ${ }^{84}$. Cs substitution allows modeling the electronic properties of those phases fairly accurately, but we stress that it is not the way to capture possible effects of the molecular orientation on the band gap. For instance, depending on the molecular orientation, a variation within about $0.14 \mathrm{eV}$ was reported in the literature ${ }^{85}$. However, forcing molecules towards some specific static orientation in the cubic or the tetragonal phase may introduce an unrealistic bias, which can be avoided considering the highly symmetric Cs cation with negligible change on the band dispersion near the electronic gap (Figure S1).

Thus, we calculated the band structure of the 2D HOP compounds with each interlayer molecule replaced by a Cs atom. Figure 2b) shows the dispersions of GAMA as an example of $2 \mathrm{D}$ confinement effect on the electronic structure. One notices a flat dispersion along the $\mathrm{W}-\mathrm{T}$ direction in reciprocal space which corresponds to the stacking axis of the alternating inorganic and organic layers in real space. Hence, little or no electronic coupling is present along that axis. The results for the band gaps are summarized in Table II. The experimental band gaps are fundamental ones corresponding to band-to-band absorption edges except for GAMA structure, whose reported value remain a rough estimate that does not indicate a clear band edge ${ }^{65}$. In all cases, the band gaps predicted by ppTB-mBJ are in nice agreement with experiment. Let us note, however, that the continuum thresholds from the experimental absorption spectra are rarely reported as the excitonic resonances are present in layered HOP. This brings some uncertainties in the experimental value of the band gap, which could further improve or worsen the agreement between experiment and theory.

The combined results of the 3D and 2D HOP have shown that the new variant of the 
TB-mBJ potential proposed in this work is very efficient for an accurate, almost quantitative, prediction of the band gap in HOP. Nevertheless, for layered HOP, longer molecular chains might bring in additional confinement thereby affecting the band gap of the structure. If ppTB-mBJ predicts too large or too small band gaps for such systems, one could resort to fixing the parameter $c$ in Eq. (2) and bypass the use of $\alpha$ and $\beta$. To facilitate the choice of $c$ in this optimization process, we designed an empirical relationship between $c$ and the interlayer distance $L$ of the 2D HOP as defined in Figure 2 ( $L=0$ would correspond to the 3D case). Figure 3 shows the evolution of $c$ and the average $\langle\nabla \rho / \rho\rangle^{1 / 2}=\left(\left(1 / V_{\text {cell }}\right) \int_{\text {cell }}\left|\nabla \rho\left(\mathbf{r}^{\prime}\right)\right| / \rho\left(\mathbf{r}^{\prime}\right) d^{3} r^{\prime}\right)^{1 / 2}$ from Eq. (2) with respect to the interlayer separation $L$, and a good fit of the data is given by:

$$
c_{\text {fit }}=2.2507-0.03376 L .
$$

We note that $c$ decreases with $L$, which can be understood through the diminishing value of the density term $\langle\nabla \rho / \rho\rangle^{1 / 2}$ as $L$ increases, which in turn is induced by the volume increase. One may view Eq. (4) as a plausible (and new) alternative to Eq. (2). Actually, Eq. (4) sheds light on the relation between three quantities; the average $\langle\nabla \rho / \rho\rangle^{1 / 2}$ in the unit cell, the interlayer separation and the band gap. Hence, Eq. (4) could serve as an alternative starting point to fine tune the TB-mBJ parameters for band gap prediction in the case ppTB-mBJ fails for a particular HOP material within the pseudopotential implementation.

\section{Effective masses}

As discussed in Section III A, ppTB-mBJ leads to band widths of the VBM and CBM in the 3D HOP that are clearly smaller than the values from LDA and possibly also experiment. Consequently, as already pointed out in ref. 82 for simple semiconductors and insulators, TB-mBJ may be inaccurate for the effective masses in HOP, which are often computed within the parabolic approximation of the band dispersion with respect to the wavevector $k$ around a high symmetry point in the Brillouin zone or within the finite difference approximation. Both approaches may be prone to errors and sophisticated, but computationally more demanding methods, may be required to alleviate problems as well as to treat more accurately degenerate bands ${ }^{89}$. Nevertheless, using the finite difference method, as used in this work, can bring some semi-quantitative information on the effect 


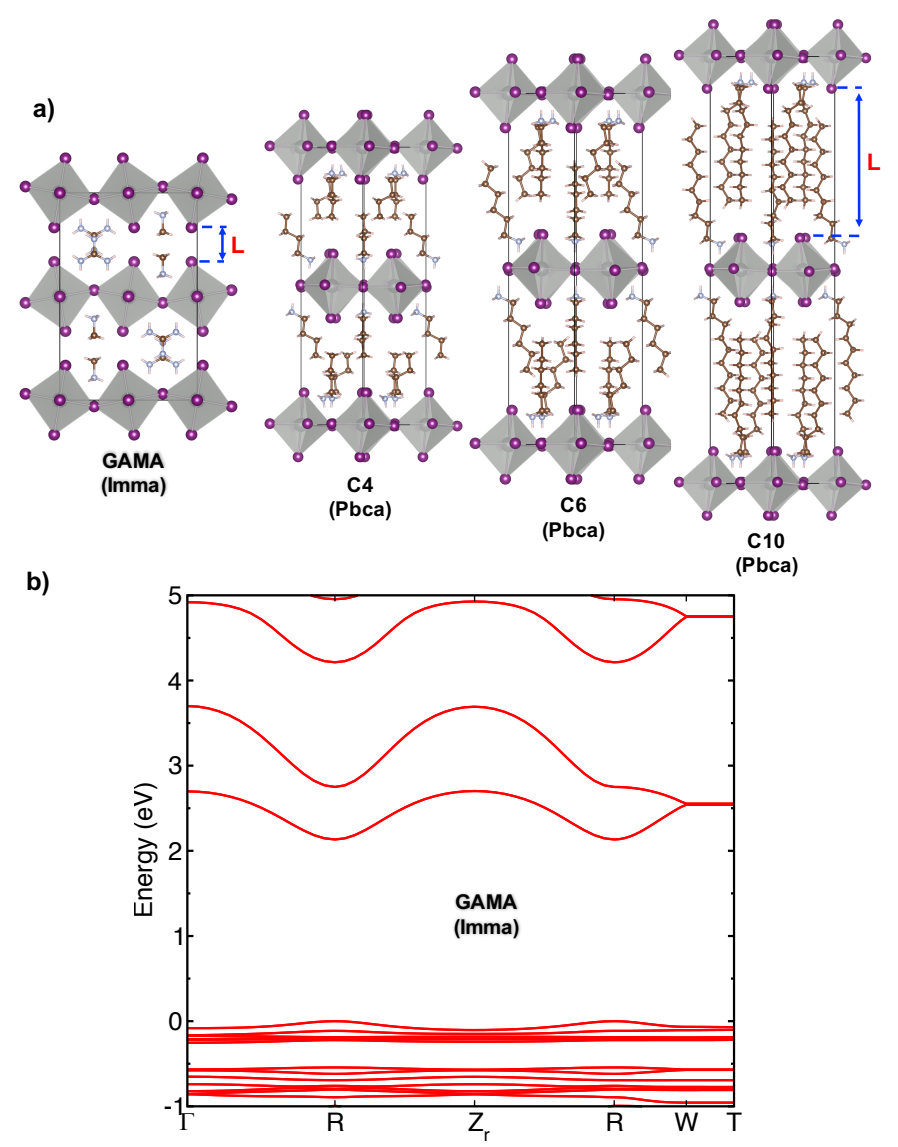

FIG. 2: a) The 2D HOP layered structures considered in this work. The space group is given in parenthesis. The distance $L$ is defined as the separation between the different layers of inorganic octahedra sandwiched between the molecular spacers. b) Band structure of the complete GAMA structure as an example of 2D confinement including the molecules. One notices a flat dispersion along $\mathrm{W}-\mathrm{T}$ direction which corresponds to the stacking axis in real space. $Z_{r}$ corresponds to $[1 / 4,1 / 4,-1 / 4]$.

of ppTB-mBJ on the effective masses of HOP compared to LDA, and Table III summarizes the LDA and ppTB-mBJ results for layered HOP. Compared to the experimental data that are available for $\mathrm{C} 4$ and $\mathrm{C} 6$ for the reduced effective mass $\mu=m_{e} m_{h} /\left(m_{e}+m_{h}\right)$, ppTBmBJ leads to errors that are of the same order of magnitude as LDA, but with opposite trends; ppTB-mBJ (LDA) overestimates (underestimates) the values by $\sim 0.1$. From the results obtained for the band widths as discussed above, such results are expected. Similar trends are obtained for the 3D compounds (Table $\mathrm{S} 1$ of the $\mathrm{SM}^{83}$ ). However, in the case of $\mathrm{MAPbI}_{3}$, for which very accurate experimental data exist, ppTB-mBJ seems to perform 
TABLE II: Band gap (in eV) of 2D HOP obtained with LDA and two reoptimized versions of TB-mBJ: jTB-mBJ from Jishi et al. ${ }^{45}$ and ppTB-mBJ proposed in the present work. The space group is indicated in parenthesis. SOC effects were included in all calculations.

\begin{tabular}{lcccc}
\hline \hline 2D HOP & LDA & jTB-mBJ & ppTB-mBJ & Exp. \\
\hline GAMA (Imma) & 0.98 & 1.76 & 2.09 & $2.27^{65 \mathrm{a}}$ \\
C4 (Pbca) & 1.13 & 2.40 & 3.01 & $2.83^{86 \mathrm{~b}}$ \\
C6 (Pbca) & 1.12 & 2.22 & 2.70 & $2.70^{87 \mathrm{c}}$ \\
C10 (Pbca) & 1.13 & 1.98 & 2.46 & $2.72^{88 \mathrm{~d}}$ \\
\hline \hline
\end{tabular}

a Measured at $293 \mathrm{~K}$.

b Measured at at room temperature.

c Measured at $5 \mathrm{~K}$.

${ }^{\mathrm{d}}$ We obtained this value by adding its exciton binding energy of $0.32 \mathrm{eV}$ to its exciton peak position $(2.4 \mathrm{eV})$ at room temperature as in ref. 88 .

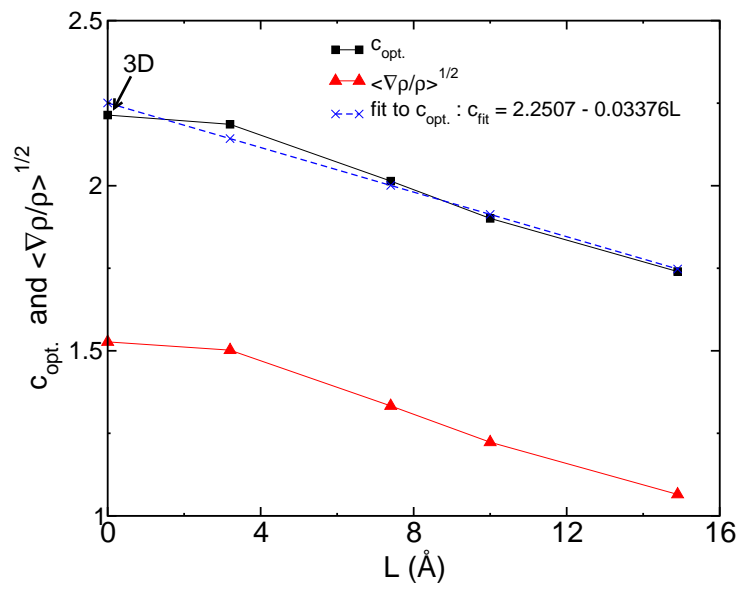

FIG. 3: Variation of $c$ and the average $\langle\nabla \rho / \rho\rangle^{1 / 2}(\mathrm{Eq}(2))$ with respect to the interlayer distance $L$ in 2D HOP structures. The values at $L=0$ correspond to 3D HOP and were calculated as the average of the different 3D structures of Table I. The values of $c$ are given in Tables S2 and S3 of the SM. 
slightly better; the overestimation due to ppTB-mBJ is in the range $0.02-0.08$ while LDA underestimates the values by at least 0.06 . The latter might be related to the very narrow bandgap obtained with LDA.

A characteristic feature of TB-mBJ is that optimizing the parameter $c$ to predict the experimental band gap inevitably leads to the overestimation of the effective masses in agreement with ref. 90. This is illustrated in Figure 4 for $\mathrm{MAPbI}_{3}$ and $\mathrm{C} 4$ structures. In other words, the same optimized $c$ cannot be expected to produce at the same the correct band gap and effective masses. In general, this ppTB-mBJ overestimation of the effective mass is inherent to the way ppTB-mBJ is forced to produce a correct band gap through the parameter $c$. As the latter increases to open the band gap, the ppTB-mBJ potential becomes more attractive which leads to more localized electrons, thus narrowing the band width of the VBM in particular. The pronounced overestimation of effective masses for layered structures is probably related to the additional confinement induced by their low-dimensionality, enhancing the TB-mBJ attrative potential. This suggests that great care should be taken if quantities other than the band gap, e.g., the effective mass or the absorption spectrum, are to be computed. 
TABLE III: Electron $\left(m_{e}\right)$ and hole $\left(m_{h}\right)$ effective masses in $m_{0}$ unit of 2D HOP calculated with the LDA and reoptimized ppTB-mBJ methods. $\mu=m_{e} m_{h} /\left(m_{e}+m_{h}\right)$ is the reduced effective mass.

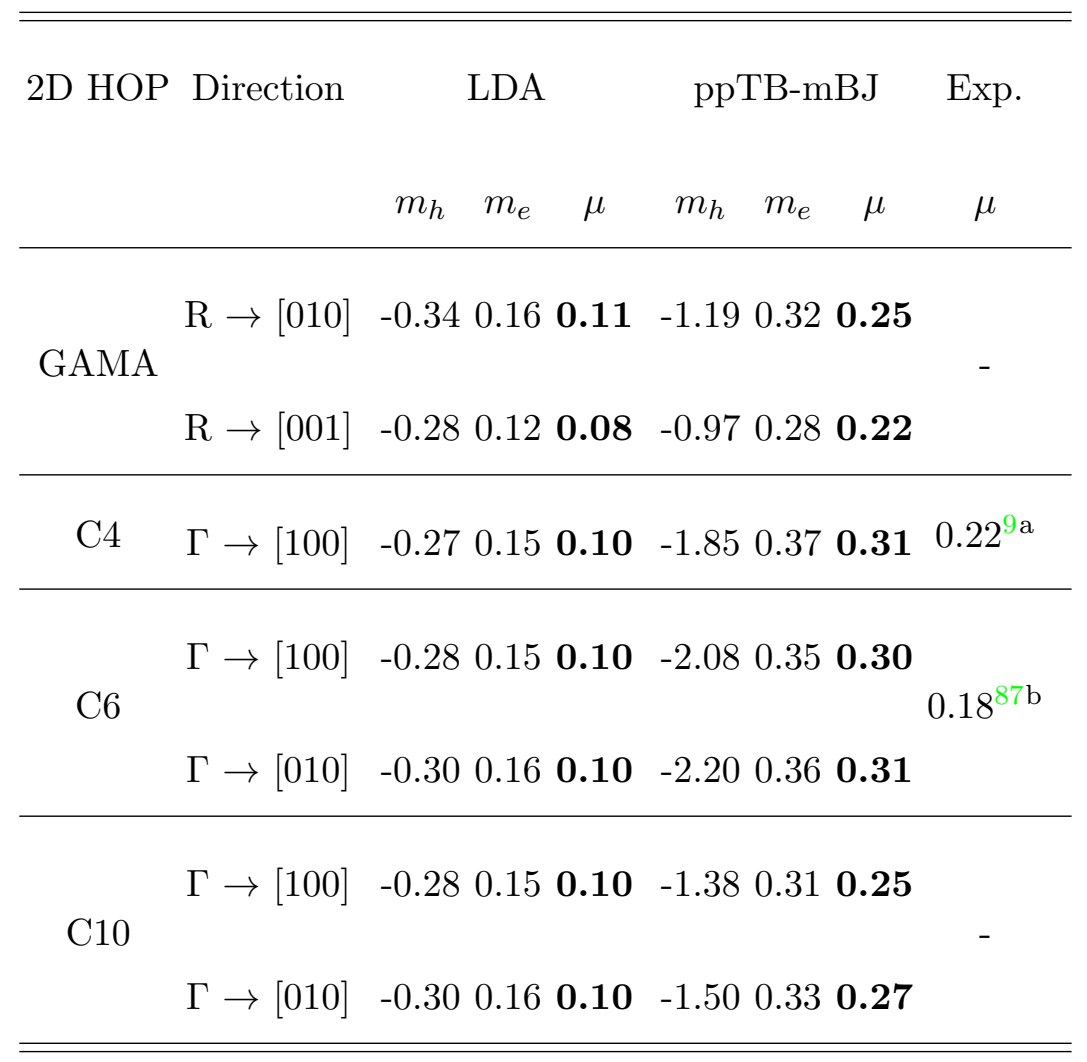

${ }^{\text {a }}$ Measured at $4 \mathrm{~K}$.

b Measured at $5 \mathrm{~K}$. 
a)

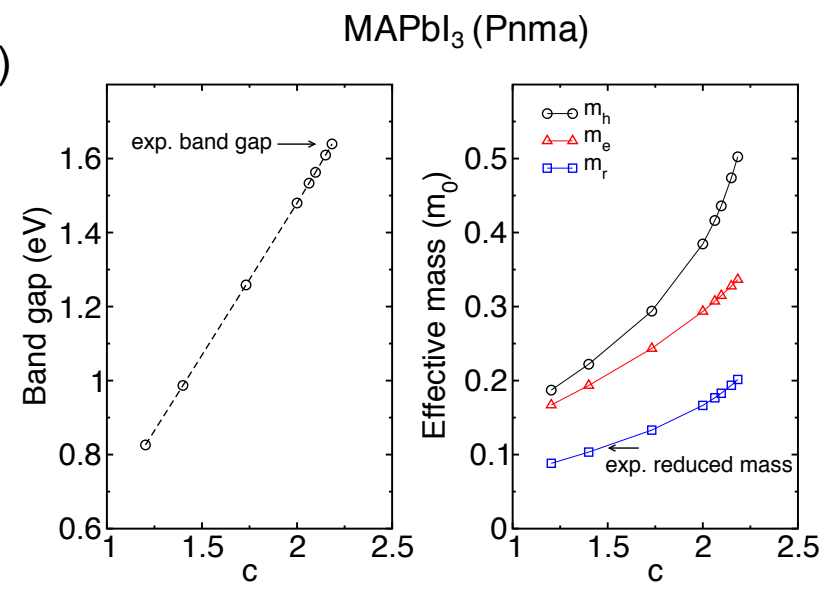

b)

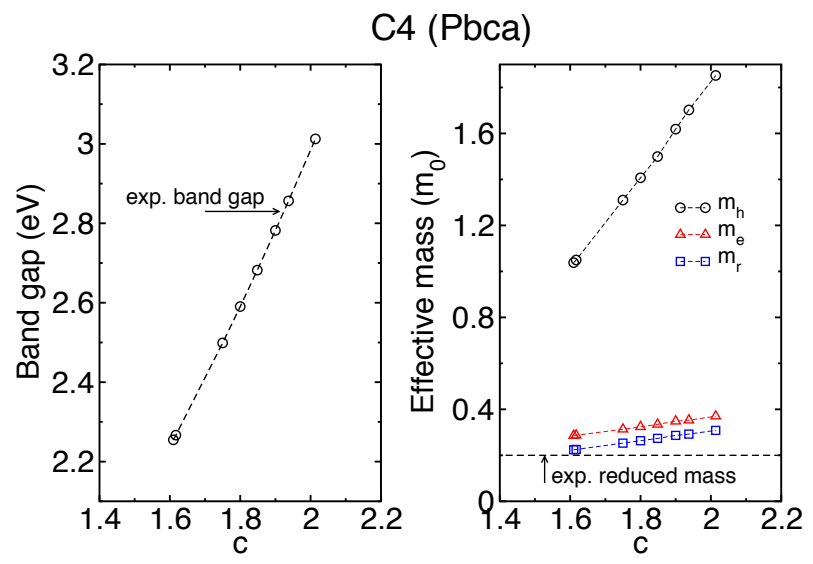

FIG. 4: Variation of the band gap and effective masses with respect to $c$ for (a) $\mathrm{MAPbI}_{3}$ (Pnma) and (b) C4 (Pbca) structures. The figure highlights the conflicting behavior of $c$ to optimize the band and the effective masses. 


\section{Pitfalls, limitations and issues}

Although the TB-mBJ potential is very appealing since reliable band gaps can be obtained at a cheap computational cost, it may present some issues which we describe in this section. First and foremost, as discussed recently in ref. 36, the main problem of the TB-mBJ potential is basically to illegally provide band gaps in good agreement with experiment. Illegally in the sense that a multiplicative potential like LDA or TB-mBJ is expected to provide a value for CBM - VBM (the usual way to calculate the band gap in KS-DFT) that differs from $I-A$ (ionization potential $I$ minus the electron affinity $A$ ) which is the definition of the true band gap. Indeed, it is known that CBM-VBM and $I-A$ differ by the exchange-correlation discontinuity ${ }^{91,92}$ which can be substantial. In other words, achieving agreement with experiment for the band gap is physically not justified, which may lead to problems for other calculated quantities.

Misplaced states within the band gap: By using the pseudopotential implementation of TB-mBJ in ABINIT, we have found that for 2D perovskites with longer organic chains, without Cs substitution, misplaced states within the band gap may appear. This is illustrated in Figure 5 for the case of $\mathrm{C} 4$, where we can observe such a (flat) band located $1 \mathrm{eV}$ above the VBM. These misplaced mid-gap states belong to the butylammonium $\left(\mathrm{BA}=\mathrm{C}_{4} \mathrm{H}_{9} \mathrm{NH}_{3}\right)$ molecule, i.e., the organic spacer. Similar results were obtained for the $\mathrm{C} 6$ HOP. However, when using the TB-mBJ implementation in the all-electron code WIEN2k (with the parametrization of Jishi et $a l_{.}{ }^{45}$ ) for the C4 structure, no such misplaced states within the band gap are present, as shown in Figure $\mathrm{S} 2$ of the $\mathrm{SM}^{83}$. The parasitic flat band obtained with ABINIT is most likely due to the used LDA pseudopotentials, which are not adapted for TB-mBJ. Thus, that means that one has to be careful when using the TB-mBJ potential within a pseudopotential-based DFT code, and this calls for the development of pseudopotentials specific to TB-mBJ. Nevertheless, substituting the molecular chain with Cs atoms solves this specific problem since no such misplaced (parasitic/artificial) bands are obtained. Hence, the Cs substitution not only reduces the computational load, which was our main objective, but also avoids the appearance of misplaced molecular states within the band gap when long chains are used. In such benchmark studies, the Cs substitution is an interesting route to predicting the band gap of layered HOP.

Nevertheless, we note that Cs substitution remains a mere conceptual way of treating 
the band structure of these large 2D systems at a much-reduced computational cost. However, for calculations related to the computation of absorption spectra using Bethe-Salpeter equation (BSE) like methods, where dielectric screening plays a crucial role, Cs substitution should not be relevant.

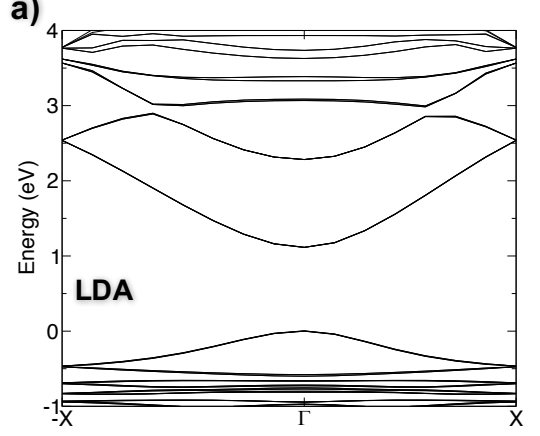

b)

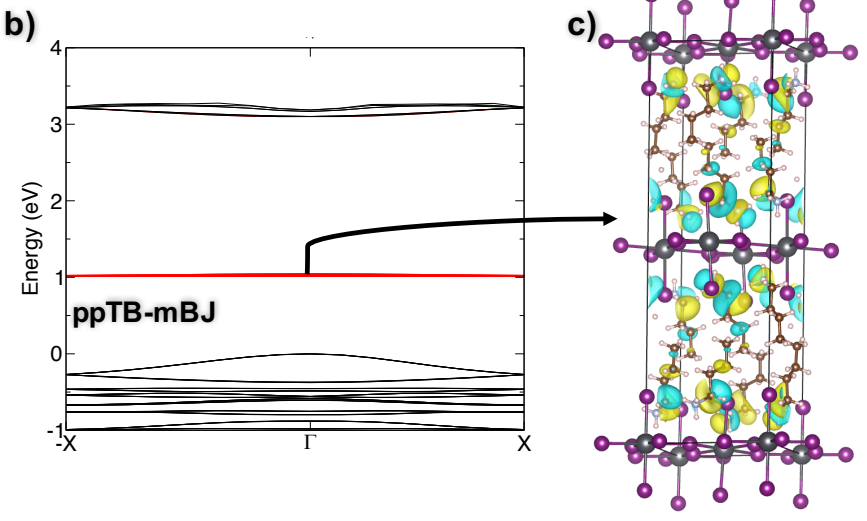

FIG. 5: Band structure of the 2D HOP C4, with the full BA molecular chain included in the calculations, obtained with a) LDA and b) ppTB-mBJ. The wave function at $\Gamma$ of the localized states within the band gap obtained with ppTB-mBJ is shown in c). These misplaced states are due to the BA molecules of the organic interlayer spacer.

Sn based 2D HOP: The ppTB-mBJ parameters that we have proposed in this work $\left(\alpha=0.65, \beta=1.023\right.$ bohr $^{1 / 2}$ ) largely overestimate the band gap for $\mathrm{BA}_{2} \mathrm{SnPbI}_{4}$. For this system, ppTB-mBJ leads to a band gap of $3.12 \mathrm{eV}$, which is much larger than the experimental value of $1.83 \mathrm{eV}^{93}$ (see the band structures in Figure $\mathrm{S} 3$ of the $\mathrm{SM}^{83}$ ). We found that agreement with experiment is reached by reducing significantly the parameter $\alpha$ to a value of 0.10 , which leads to a band gap of $1.85 \mathrm{eV}$. Hence, unlike for the $\mathrm{Pb}$ and $\mathrm{Sn} 3 \mathrm{D}$ HOP and $\mathrm{Pb} 2 \mathrm{D}$ HOP, this 2D HOP with $\mathrm{Sn}$ as the divalent metal cation requires different TB-mBJ parameters for reliable band gap predictions. Let us note that despite the generality of our TB-mBJ approach, we speculate that our optimized parameters work best for Pb-based hybrid halide perovskites. For systems using different metal B cations such as in double perovskites or Sn in 2D systems (vide supra), different TB-mBJ parameters may be needed for accurate band gap prediction. 


\section{E. Conclusions}

In this contribution, we have reoptimized the parameters in the TB-mBJ potential for 3D and 2D layered HOP within a pseudpotential-based implementation of DFT. The reoptimized parameters predict band gaps that are in fair agreement with experiment. Noteworthy, TB-mBJ overestimates the effective masses due to the narrowing of the band dispersions, especially for the valence bands. This means that great care should be taken if quantities other than the band gap are of interest. Our study also demonstrates that substituting long molecular chains of the 2D layered structures with Cs atoms is an interesting and costeffective route towards predicting band gaps in general. Importantly, we propose a scheme to address the band gap problem of HOP with the TB-mBJ potential, which could be extended to other layered materials.

Nevertheless, we note that our ppTB-mBJ parameters work best for $\mathrm{Pb}$ based and to some extent for 3D Sn based perovskites. Our optimized parameters might not be suitable for double perovskites and perovskite systems, whose octahedral environments significantly differ from those of $\mathrm{Pb}$-based HOP. We believe that the strategy implemented in the present work is a good starting point to optimize TB-mBJ parameters for other families of halide perovskites such as double perovskites. Furthermore, the effect of electron-phonon coupling ${ }^{94}$ is not considered in this work, which may bring additional changes to the predicted band gaps especially for cubic HOP compounds ${ }^{68}$.

Overall, TB-mBJ provides an efficient and computationally cheap framework for band gap prediction and is an appealing alternative to hybrid functionals and the $G W$ method for large systems such as hybrid organic-inorganic perovskites.

\section{ACKNOWLDGEMENT}

The work at the Institut des Sciences Chimiques de Rennes was supported by Agence Nationale pour la Recherche (TRANSHYPERO project). This work was granted access to the HPC resources of [TGCC/CINES/IDRIS] under the allocation 2017-A0010907682 made by GENCI. ISCR have received funding from the European Union's Horizon 2020 program, through a FET Open research and innovation action under the grant agreement No 687008. F.T. acknowledges support from the Austrian Science Fund (FWF) through the project 
B. Traore et coll. Phys. Rev. B 2019; https://journals.aps.org/prb/accepted/

ad07406bJf21963f606384008ec0d981852de2b59

F41 (SFB ViCoM). The authors thank Prof. Jacky Even and Dr. Laurent Pedesseau from INSA/FOTON.

* boubacar.traore@univ-rennes1.fr

1 A. Kojima, K. Teshima, Y. Shirai, and T. Miyasaka, J. Am. Chem. Soc. 131, 6050 (2009).

2 https://www.nrel.gov/pv/assets/images/efficiency-chart.png, accessed: 2018-07-03.

3 S. Adjokatse, H.-H. Fang, and M. A. Loi, Mater Today 20, 413 (2017).

4 Y.-H. Kim, H. Cho, and T.-W. Lee, Proc. Natl. Acad. Sci. USA 113, 11694 (2016).

5 S. Yakunin, M. Sytnyk, D. Kriegner, S. Shrestha, M. Richter, G. J. Matt, H. Azimi, C. J. Brabec, J. Stangl, M. V. Kovalenko, and W. Heiss, Nat. Photonics 9, 444 (2015).

6 G. Xing, N. Mathews, S. S. Lim, N. Yantara, X. Liu, D. Sabba, M. Grätzel, S. Mhaisalkar, and T. C. Sum, Nat. Materials 13, 476 (2014).

7 I. C. Smith, E. T. Hoke, D. Solis-Ibarra, M. D. McGehee, and H. I. Karunadasa, Angew. Chem., Int. Ed. 53, 11232 (2014).

8 H. Tsai, W. Nie, J. Blancon, C. Stoumpos, R. Asadpour, B. Harutyunyan, A. Neukirch, R. Verduzco, J. Crochet, S. Tretiak, L. Pedesseau, J. Even, M. Alam, G. Gupta, J. Lou, P. Ajayan, M. Bedzyk, M. Kanatzidis, and A. Mohite, Nature 536, 312 (2016).

9 J.-C. Blancon, A. V. Stier, H. Tsai, W. Nie, C. C. Stoumpos, B. Traoré, L. Pedesseau, M. Kepenekian, F. Katsutani, G. T. Noe, J. Kono, S. Tretiak, S. A. Crooker, C. Katan, M. G. Kanatzidis, J. J. Crochet, J. Even, and A. D. Mohite, Nat. Commun. 9, 2254 (2018).

10 Y. Kato, D. Ichii, K. Ohashi, H. Kunugita, K. Ema, K. Tanaka, T. Takahashi, and T. Kondo, Solid State Commun. 128, 15 (2003).

11 M. Kumagai and T. Takagahara, Phys. Rev. B 40, 12359 (1989).

12 T. Ishihara, J. Takahashi, and T. Goto, Solid State Commun. 69, 933 (1989).

13 P. Hohenberg and W. Kohn, Phys. Rev. 136, B864 (1964).

14 W. Kohn and L. J. Sham, Phys. Rev. 140, A1133 (1965).

15 A. D. Becke, Phys. Rev. A 38, 3098 (1988).

16 J. P. Perdew, K. Burke, and M. Ernzerhof, Phys. Rev. Lett. 77, 3865 (1996).

17 E. Mosconi, C. Quarti, and F. De Angelis, "Chapter 8 first principles modeling of perovskite solar cells: Interplay of structural, electronic and dynamical effects," in Unconventional Thin 
Film Photovoltaics (RSC, 2016) pp. 234-296.

18 J. Even and C. Katan, "Chapter 1.2 ab-initio and first principles studies of halide perovskites," in Halide Perovskites: Photovoltaics, Light Emitting Devices and Beyond (Wiley-VCH, 2018).

19 J. Heyd, J. E. Peralta, G. E. Scuseria, and R. L. Martin, J. Chem. Phys. 123, 174101 (2005).

20 C. J. Cramer and D. G. Truhlar, Phys. Chem. Chem. Phys. 11, 10757 (2009).

21 J. Even, L. Pedesseau, J.-M. Jancu, and C. Katan, J. Phys. Chem. Lett. 4, 2999 (2013).

22 A. D. Becke, J. Chem. Phys. 98, 5648 (1993).

23 J. P. Perdew, M. Ernzerhof, and K. Burke, J. Chem. Phys. 105, 9982 (1996).

24 J. Heyd, G. E. Scuseria, and M. Ernzerhof, J. Chem. Phys. 118, 8207 (2003).

25 J. Heyd, G. E. Scuseria, and M. Ernzerhof, J. Chem. Phys. 124, 219906 (2006).

26 L. Hedin, Phys. Rev. 139, A796 (1965).

27 M. S. Hybertsen and S. G. Louie, Phys. Rev. B 34, 5390 (1986).

28 E. Menéndez-Proupin, P. Palacios, P. Wahnón, and J. C. Conesa, Phys. Rev. B 90, 045207 (2014).

29 J. Feng and B. Xiao, J. Phys. Chem. C 118, 19655 (2014).

30 P. Umari, E. Mosconi, and F. De Angelis, Sci. Rep. 4, 4467 (2014).

31 J. Even, L. Pedesseau, J.-M. Jancu, and C. Katan, Phys. Status Solidi RRL 8, 31 (2014).

32 M. R. Filip, C. Verdi, and F. Giustino, J. Phys. Chem. C 119, 25209 (2015).

33 E. Mosconi, P. Umari, and F. De Angelis, Phys. Chem. Chem. Phys. 18, 27158 (2016).

34 A. Fraccarollo, V. Cantatore, G. Boschetto, L. Marchese, and M. Cossi, J. Chem. Phys. 144, 164701 (2016).

35 F. Tran and P. Blaha, J. Phys. Chem. A 121, 3318 (2017).

36 F. Tran, S. Ehsan, and P. Blaha, Phys. Rev. Materials 2, 023802 (2018).

37 F. Tran and P. Blaha, Phys. Rev. Lett. 102, 226401 (2009).

38 D. Koller, F. Tran, and P. Blaha, Phys. Rev. B 83, 195134 (2011).

39 H. Jiang, J. Chem. Phys. 138, 134115 (2013).

40 K. Nakano and T. Sakai, J. Appl. Phys. 123, 015104 (2018).

41 A. D. Becke and E. R. Johnson, J. Chem. Phys. 124, 221101 (2006).

42 S. Goedecker, M. Teter, and J. Hutter, Phys. Rev. B 54, 1703 (1996).

43 J. P. Perdew and Y. Wang, Phys. Rev. B 45, 13244 (1992).

44 A. D. Becke and M. R. Roussel, Phys. Rev. A 39, 3761 (1989). 
45 R. A. Jishi, O. B. Ta, and A. A. Sharif, J. Phys. Chem. C 118, 28344 (2014).

46 P. Blaha, K. Schwarz, G. K. H. Madsen, D. Kvasnicka, J. Luitz, R. Laskowski, F. Tran, and L. D. Marks, WIEN2K: An Augmented Plane Wave plus Local Orbitals Program for Calculating Crystal Properties (Vienna University of Technology, Austria, 2018).

47 D. Koller, F. Tran, and P. Blaha, Phys. Rev. B 85, 155109 (2012).

48 X. Gonze, B. Amadon, P.-M. Anglade, J.-M. Beuken, F. Bottin, P. Boulanger, F. Bruneval, D. Caliste, R. Caracas, M. Côté, T. Deutsch, L. Genovese, P. Ghosez, M. Giantomassi, S. Goedecker, D. Hamann, P. Hermet, F. Jollet, G. Jomard, S. Leroux, M. Mancini, S. Mazevet, M. Oliveira, G. Onida, Y. Pouillon, T. Rangel, G.-M. Rignanese, D. Sangalli, R. Shaltaf, M. Torrent, M. Verstraete, G. Zerah, and J. Zwanziger, Comput. Phys. Commun. 180, 2582 (2009). 49 J. Even, L. Pedesseau, and C. Katan, ChemPhysChem 15, 3733 (2014).

50 C. Katan, L. Pedesseau, M. Kepenekian, A. Rolland, and J. Even, J. Mater. Chem. A 3, 9232 (2015).

51 B. Traore, L. Pedesseau, L. Assam, X. Che, J.-C. Blancon, H. Tsai, W. Nie, C. C. Stoumpos, M. G. Kanatzidis, S. Tretiak, A. D. Mohite, J. Even, M. Kepenekian, and C. Katan, ACS Nano 12, 3321 (2018).

52 C. Hartwigsen, S. Goedecker, and J. Hutter, Phys. Rev. B 58, 3641 (1998).

53 O. K. Andersen, Phys. Rev. B 12, 3060 (1975).

54 D. J. Singh and L. Nordström, Planewaves, Pseudopotentials, and the LAPW Method, 2nd ed. (Springer, New York, 2006).

55 M. A. Marques, M. J. Oliveira, and T. Burnus, Comput. Phys. Commun. 183, 2272 (2012).

56 Y. Kawamura, H. Mashiyama, and K. Hasebe, J. Phys. Soc. Jpn. 71, 1694 (2002).

57 T. Baikie, Y. Fang, J. M. Kadro, M. Schreyer, F. Wei, S. G. Mhaisalkar, M. Grätzel, and T. J. White, J. Mater. Chem. A 1, 5628 (2013).

58 G. A. Elbaz, D. B. Straus, O. E. Semonin, T. D. Hull, D. W. Paley, P. Kim, J. S. Owen, C. R. Kagan, and X. Roy, Nano Lett. 17, 1727 (2017).

59 I. Swainson, R. Hammond, C. Soullière, O. Knop, and W. Massa, J. Solid State Chem. 176, 97 (2003).

60 D. Trots and S. Myagkota, J. Phys. Chem. Solids 69, 2520 (2008).

61 C. C. Stoumpos, C. D. Malliakas, J. A. Peters, Z. Liu, M. Sebastian, J. Im, T. C. Chasapis, A. C. Wibowo, D. Y. Chung, A. J. Freeman, B. W. Wessels, and M. G. Kanatzidis, Cryst. 
Growth Des. 13, 2722 (2013).

62 R. L. Moreira and A. Dias, J Phys. Chem Solids 68, 1617 (2007).

63 C. C. Stoumpos, C. D. Malliakas, and M. G. Kanatzidis, Inorg. Chem. 52, 9019 (2013).

64 I. Chung, J.-H. Song, J. Im, J. Androulakis, C. D. Malliakas, H. Li, A. J. Freeman, J. T. Kenney, and M. G. Kanatzidis, J Am. Chem. Soc. 134, 8579 (2012).

65 C. M. M. Soe, C. C. Stoumpos, M. Kepenekian, B. Traoré, H. Tsai, W. Nie, B. Wang, C. Katan, R. Seshadri, A. D. Mohite, J. Even, T. J. Marks, and M. G. Kanatzidis, J. Am. Chem. Soc. 139, 16297 (2017).

66 D. G. Billing and A. Lemmerer, Acta Cryst. B 63, 735 (2007).

67 A. Lemmerer and D. G. Billing, Dalton Trans. 41, 1146 (2012).

68 C. E. Patrick, K. W. Jacobsen, and K. S. Thygesen, Phys. Rev. B 92, 201205 (2015).

69 J. Wiktor, U. Rothlisberger, and A. Pasquarello, J. Phys. Chem. Lett. 8, 5507 (2017).

70 R. Saran, A. Heuer-Jungemann, A. G. Kanaras, and R. J. Curry, Adv. Optical Mater. 5, 1700231 (2017).

71 W. Huang, S. Yue, Y. Liu, L. Zhu, P. Jin, Q. Wu, Y. Zhang, Y. Chen, K. Liu, P. Liang, S. Qu, Z. Wang, and Y. Chen, ACS Photonics 5, 1583 (2018).

72 Y. Dang, Y. Liu, Y. Sun, D. Yuan, X. Liu, W. Lu, G. Liu, H. Xia, and X. Tao, CrystEngComm 17,665 (2015).

73 G. E. Eperon, S. D. Stranks, C. Menelaou, M. B. Johnston, L. M. Herz, and H. J. Snaith, Energy Environ. Sci. 7, 982 (2014).

74 D. Shi, V. Adinolfi, R. Comin, M. Yuan, E. Alarousu, A. Buin, Y. Chen, S. Hoogland, A. Rothenberger, K. Katsiev, Y. Losovyj, X. Zhang, P. A. Dowben, O. F. Mohammed, E. H. Sargent, and O. M. Bakr, Science 347, 519 (2015).

75 Q. Dong, Y. Fang, Y. Shao, P. Mulligan, J. Qiu, L. Cao, and J. Huang, Science 347, 967 (2015).

76 C. Quarti, E. Mosconi, J. M. Ball, V. D’Innocenzo, C. Tao, S. Pathak, H. J. Snaith, A. Petrozza, and F. De Angelis, Energy Environ. Sci. 9, 155 (2016).

77 M. I. Saidaminov, A. L. Abdelhady, B. Murali, E. Alarousu, V. M. Burlakov, W. Peng, I. Dursun, L. Wang, Y. He, G. Maculan, A. Goriely, T. Wu, O. F. Mohammed, and O. M. Bakr, Nat. Commun. 6, 7586 (2015).

78 J. H. Noh, S. H. Im, J. H. Heo, T. N. Mandal, and S. I. Seok, Nano Letters 13, 1764 (2013). 
79 O. N. Yunakova, V. K. Miloslavskii, and E. N. Kovalenko, Opt. Spectrosc. 112, 91 (2012).

80 Z. Liu, J. A. Peters, C. C. Stoumpos, M. Sebastian, B. W. Wessels, J. Im, A. J. Freeman, and M. G. Kanatzidis, in Proc. SPIE, Vol. 8852 (2013) p. 88520A.

81 O. N. Yunakova, V. K. Miloslavskii, E. N. Kovalenko, and E. V. Ksenofontova, Low Temp. Phys. 38, 943 (2012).

82 D. Waroquiers, A. Lherbier, A. Miglio, M. Stankovski, S. Poncé, M. J. T. Oliveira, M. Giantomassi, G.-M. Rignanese, and X. Gonze, Phys. Rev. B 87, 075121 (2013).

83 See Supplemental Material at [URL will be inserted by publisher] for : Validity of substituting interlayer molecules by Cs atoms for states close to the band gap; Computed effective masses for 3D HOP; Band structure of $\mathrm{BA}_{2} \mathrm{PbI}_{4}$ with WIEN2k; Band structure of $\mathrm{BA}_{2} \mathrm{SnI}_{4}$; Values of $c$ obtained from $\alpha=0.65, \beta=1.023 \mathrm{bohr}^{1 / 2}$ for both 3D and 2D HOP.

84 M. A. Carignano, S. A. Aravindh, I. S. Roqan, J. Even, and C. Katan, J. Phys. Chem. C 121, 20729 (2017).

85 C. Quarti, E. Mosconi, and F. De Angelis, Chem. Mater. 26, 6557 (2014).

86 J.-C. Blancon, H. Tsai, W. Nie, C. C. Stoumpos, L. Pedesseau, C. Katan, M. Kepenekian, C. M. M. Soe, K. Appavoo, M. Y. Sfeir, S. Tretiak, P. M. Ajayan, M. G. Kanatzidis, J. Even, J. J. Crochet, and A. D. Mohite, Science 335, 1288 (2017).

87 K. Tanaka, T. Takahashi, T. Kondo, T. Umebayashi, K. Asai, and K. Ema, Phys. Rev. B 71, $045312(2005)$.

88 T. Ishihara, J. Takahashi, and T. Goto, Phys. Rev. B 42, 11099 (1990).

89 N. A. Mecholsky, L. Resca, I. L. Pegg, and M. Fornari, Phys. Rev. B 89, 155131 (2014).

90 Y.-S. Kim, M. Marsman, G. Kresse, F. Tran, and P. Blaha, Phys. Rev. B 82, 205212 (2010).

91 J. P. Perdew, R. G. Parr, M. Levy, and J. L. Balduz, Jr., Phys. Rev. Lett. 49, 1691 (1982).

92 L. J. Sham and M. Schlüter, Phys. Rev. Lett. 51, 1888 (1983).

93 D. H. Cao, C. C. Stoumpos, T. Yokoyama, J. L. Logsdon, T.-B. Song, O. K. Farha, M. R. Wasielewski, J. T. Hupp, and M. G. Kanatzidis, ACS Energy Lett. 2, 982 (2017).

94 M. Zacharias and F. Giustino, Phys. Rev. B 94, 075125 (2016). 